\title{
IUSE: EHR - Enhancing and Expanding Experiential Learning Modules across Disciplines and Institutions
}

\section{Dr. Gloria J. Kim, Northwestern University}

Gloria Kim is an Associate Professor of Instruction in the Department of Biomedical Engineering at Northwestern University. She also a courtesy faculty member with the Department of Electrical and Computer Engineering at the University of Florida. She obtained her B.S. in Chemistry from Seoul National University, M.S. in Biomedical Engineering from Johns Hopkins University, and Ph.D. in Biomedical Engineering from Georgia Institute of Technology. She teaches courses in biomechanics, biomaterials, bioinstrumentation, and nanotechnology.

\section{Dr. Robert Linsenmeier, Northwestern University}

Robert Linsenmeier is a Professor of Biomedical Engineering, Neurobiology, and Ophthalmology. His interests are in the microenvironment of the mammalian retina and engineering education. His teaching is primarily in physiology for both biology and BME majors. He is a fellow if the Biomedical Engineering Society, American Institute of Medical and Biological Engineering, and Association for Research in Vision and Ophthalmology. He is the co-leader of CIRTL at Northwestern and Director of the Northwestern Center for Engineering Education Research.

\section{Dr. Timothy Reissman, University of Dayton}

Dr. Timothy Reissman is an Assistant Professor within the Department of Mechanical and Aerospace Engineering at the University of Dayton. He teaches primarily courses related to experimentation, mechatronics, and dynamic systems and controls.

Dr. Mary Beth Finch, Northwestern University 


\section{IUSE: EHR - Enhancing and Expanding Experiential Learning Modules across Disciplines and Institutions}

introduction

Over its NSF-funded lifetime (1998-2008) the VaNTH (Vanderbilt-Northwestern-TexasHarvard/MIT) Engineering Research Center in Bioengineering Educational Technologies showed in its many studies aspects of educational environments that could best promote student learning. With a base of ideas summarized in the book "How People Learn (HPL)" [1], VaNTH extended HPL's research base from K-12 education to undergraduate and graduate learning in bioengineering $[2,3]$. The four thrusts of HPL are: learner-centered, knowledge-centered, assessment-centered, and community-centered education. The HPL framework only highlights the need for these critical dimensions, and does not provide specific details for how to systematically design instruction to optimize them. VaNTH researchers found challenge-based instruction $(\mathrm{CBI})$ to be an effective instructional model for designing learning environments [4, 5]. Challenges provided students with the conditions for when and how to apply specific knowledge, and were particularly important for meeting more complex learning objectives [6]. Based on HPL and CBI, VaNTH produced a large number of interactive courseware modules in biomechanics [7, 8], bioinstrumentation [9], systems physiology [10, 11, 12], design [13, 14], biosignal analysis $[15,16]$, biotechnology $[17,18]$, and biomedical imaging $[18,19]$.

For this NSF-funded project (2015 - present) in the Engaged Student Learning track of Improving Undergraduate STEM Education (IUSE), we proposed to enhance and expand experiential learning modules in the domains of biosignals and systems analysis and experimental design. There were two reasons behind this choice. First, most fundamental courses in several engineering majors offered during the middle years cover concepts that fall in those domains [20]. Second, these areas were rated highly among the seventeen engineering, biology, and physiology domains by representatives in academia and industry in the VaNTH Key Content Survey that focused on identifying key concepts that all undergraduate biomedical engineers should know upon graduation [21, 22].

This executive summary provides a synopsis of the study goals, assessment methods, and how data were collected analyzed during the first two years (Fall 2015 - Spring 2017) of the threeyear project.

objectives and scope

The overall objective of this exploratory collaborative project between two institutions is to support the adaptation, implementation, and dissemination of best practices in experiential learning. Specifically, the project focuses on a three-quarter course sequence at Northwestern University's Department of Biomedical Engineering (BME305, BME306, and BME307) and a two-semester course sequence at University of Florida's Department of Electrical and Computer Engineering (EEL3111C: Circuits I and EEL4930: Modeling and Analysis of Bioelectrical Systems). Students typically start these sequences in their junior year. Both course sequences teach similar topics (circuits, signals and systems, modeling, instrumentation) that are geared towards building quantitative and computational skills needed to be successful in the workplace 
or graduate school. Both of these course sequences employ portable lab kits (Table 1) - nScope [23] at Northwestern University and Digilent Analog Discovery Scope [24] at University of Florida - that can be set up anywhere on a laptop.

Our key activities would consist of: 1) assessing the strengths and weaknesses of Northwestern University's three-course sequence in terms of the HPL framework and assessing how student experiences and outcomes in this sequence are linked to those attributes of the course; and 2) expanding experiential modules for broader adoption and implementation.

Table 1: nScope and Digilent Analog Discovery Scope Comparison

\begin{tabular}{|c|c|c|}
\hline Product & nScope & Digilent Analog Discovery Scope \\
\hline Features & $\begin{array}{l}\text { - } \\
\text { 4-channel } 4 \text { MPPS 12-bit single- } \\
\text { ended input oscilloscope } \\
\text { - } \quad \text { 2-channel waveform generator } \\
\text { (PWMannel pulse-width-modulated } \\
\text { - } \quad \pm 5 \text { VDC powerator } \\
-\quad \text { Overcurrent protection and } \\
\text { notification }\end{array}$ & $\begin{array}{ll}\text { - } & \text { 2-channel } 100 \text { MSPS 14-bit } \\
& \text { differential input oscilloscope } \\
\text { - } & \text { 2-channel waveform generator } \\
\text { - } & \text { 16-channel logic analyzer } \\
\text { - } & \text { 16-channel digital pattern generator } \\
\text { - } & \pm 5 \mathrm{VDC} \text { power supplies }\end{array}$ \\
\hline MATLAB Support & Yes & Yes \\
\hline API Support & Yes & Yes \\
\hline $\begin{array}{ll}\text { Operating } & \text { Systems } \\
\text { Supported } & \end{array}$ & Windows, Mac, Linux, ARM, Raspbian & Windows, Mac, Linux, ARM \\
\hline Academic Pricing & $\$ 69$ & $\$ 159$ \\
\hline Retail Pricing & $\$ 89$ & $\$ 259$ \\
\hline
\end{tabular}

first two years - goals and methods

The goal during the first two years of the project (Fall 2015 - Spring 2017) was to evaluate the strengths and weaknesses of Northwestern University's three-course sequence in terms of the HPL "centers," and assess how student experiences and outcomes in this sequence are linked to those attributes of the course. The knowledge would then guide future design or development of new interventions and their dissemination strategies during the third year of the project.

We employed the mixed method to evaluate learning of course concepts, learning preferences, engagement, application of material in subsequent courses in the sequence, and transferrable skills between courses. 
The quantitative study involved assessment of the student performance and attitudes as well as assessment of classroom dynamics. On the student side, we used two kinds of surveys, course evaluations and our own online survey instrument. The course evaluations gave us the opportunity to obtain a measure of student perception of the course, the instructor, the workload, and the amount learned for each course in the sequence. Additionally, we constructed and used an additional online survey instrument each quarter designed to determine the degree to which the students feel that each of the learning objectives of the courses have been accomplished, their confidence in taking on new challenges in these domains, and the degree to which they feel that the HPL centers entered into the course. This last topic was addressed by a series of elements not directly asking about HPL, but framed to reveal this. For example, to understand whether the course was assessment-centered we asked their level of agreement with the statements, "I received adequate feedback on my work," and "I was able to obtain enough practice on important topics."

Another part of the quantitative study was gauging to what extent the teaching reflects HPL. We expected simply by using the laboratory kits and employing active learning, these courses will be assessment and knowledge centered to some extent. However, observing selected classes in each course would give a better understanding of how time is spent in class, how the instructor attempts to connect new knowledge to the students' existing frameworks, and to what extent elements of the community center (e.g. opportunities for peer learning, cultural awareness, support for all students) exist. We utilized the Classroom Observation Protocol for Undergraduate STEM (COPUS) [25].

The qualitative study consisted of semi-structured interviews with the student and the instructor at different points during the course. Many of the experiential modules in the sequence share a trait with a typical design course - they are ill-structured. Therefore, students were required to become resourceful and draw from earlier courses and experiences to complete them. We documented the students' perspective on the course activities in term of understandings of what they are doing, why they are doing it, and what they learned from the activity. Instructor interviews provided us with their understandings of the purpose of course activities. These were compared with those of the students. This gave us an insight on how well the understandings of the constituents are aligned.

data analysis and results

Online survey instrument questions were mapped onto the four HPL centers. For example, if a student responded positively to "helped me connect key ideas between this course and ideas in other BME courses," this was recorded as a "Knowledge-Centered Strength" of the course. On the other hand, a negative response to "content of tests was related to the material covered in class" was recorded as a "Assessment-Centered Weakness." Conceivably, not all the survey questions could be categorized exclusively into one single HPL center. For instance, "not enough interaction / opportunities to participate" was regarded as a weakness in both learner-centered and community-centered. The percentage of strengths and weaknesses in each HPL category was plotted for each course and each instructor to measure how much three-course sequence exhibited HPL. A sample result from this analysis is shown in Figure 1. 
Items we assigned to various HPL centers included items that are instructor-specific and items that apply to the course overall. For courses taught by more than one instructor - four of the six courses we collected data from were taught by two instructors, two by design, two under extenuating circumstances - we addressed the potentially confounding factor as follows: To focus on how much teaching echoes HPL, as we have in Figure 1, we repeated the numbers for items that applied to the course overall. For example, we included course-specific items such as "tests indicated how well I understood the material I learned in class" for the score for knowledge-centeredness for both instructors. Thus, the difference in the instructors overall scores revealed the difference in the responses to instructor-specific questions. To create an overall number for the course to measure its HPL reflection, we averaged the scores for the instructor-specific questions. In that case, we averaged the responses for each instructor for items such as "helped me understand important concepts" to generate an overall score.

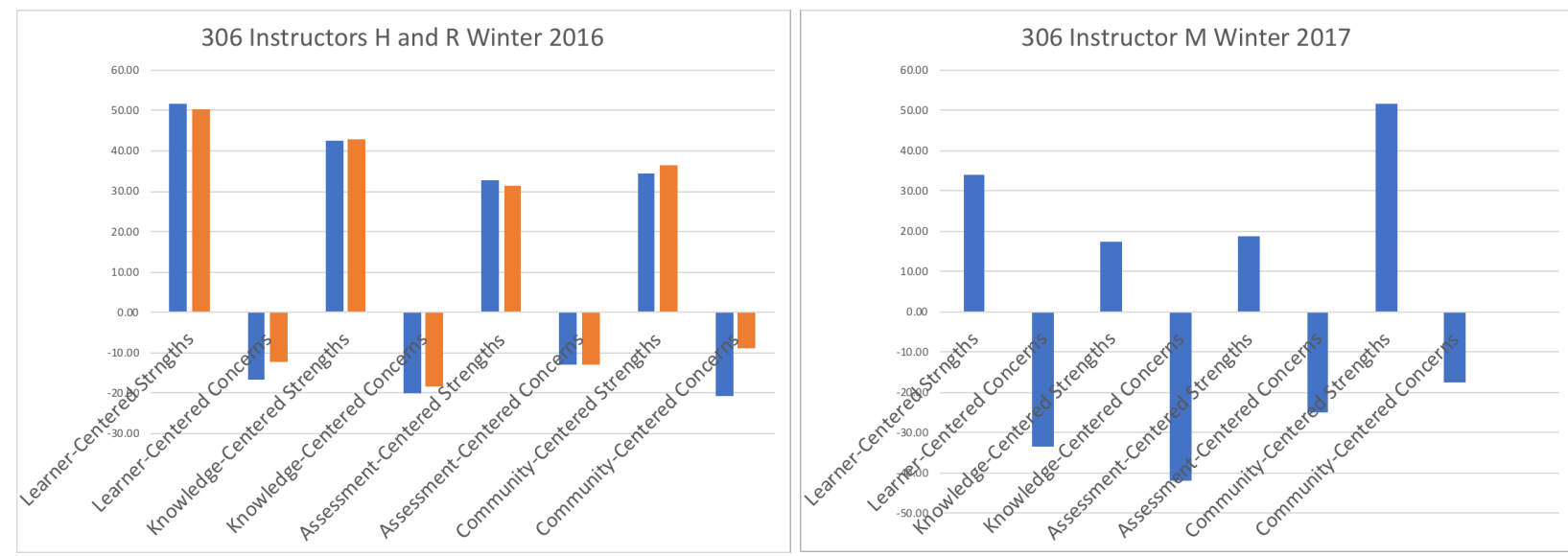

Figure 1. HPL Scores for BME 306 Instructors

We are currently analyzing COPUS and interview data. One of the interesting findings is the realization that qualitatively, trying harder to implement the HPL centers does not necessarily lead to student satisfaction with the course. This may be attributed to the fact that to date, there is no suitable tool available to translate the different viewpoints of the students and the instructors revealed in the survey/interview/observation results to HPL centers. The development of such tool is outside the scope of this exploratory project. However, such effort is warranted and expected to make a significant contribution to providing meaningful HPL-based guidelines as to how to restructure a course once its strengths and weaknesses have been identified.

next steps

With the strengths and weaknesses identified, the next part of the study is to enhance and expand effective experiential learning modules for broader adoption and implementation. Our collaborator at University of Florida has not yet fully integrated Digilent Analog Discovery Scope in their course. Since their course, EEL3111C, is also required for BME students, the portable laboratory modules at University of Florida ideally should reinforce underlying ECE concepts while providing examples of ECE and BME application. Our 305's portable modules for nScope, strike this balance. Since nScope and Digilent Analog Discovery share many similarities, we anticipate the earliest partial implantation of these modules in EEL3111C to take place in Spring 2018. The researchers will have then have the opportunity to study the way 
instructors employ new materials, the difficulties they face, and what can be done to overcome those barriers.

Perhaps the most challenging HPL principle to implement in a web-based platform is "assessment-centered," as it entails optimizing the amount and type of formative feedback provided to the students. We will use the results to examine the effectiveness of the feedback we have provided in the past and devise a strategy for effective feedback delivery in an adaptive web-based learning module.

In the long term, we expect knowledge and educational products generated from our work in the domains of biosignals and systems analysis and experimental design will benefit curricula in other areas of engineering as well, since most fundamental courses in several engineering majors offered during the middle years cover concepts that fall in those areas. From the resource point of view, the far-reaching potential of the portable lab modules and interactive/adaptive webbased learning modules is readily conceivable. 